\title{
Socio-cognitive determinants of colorectal cancer screening uptake: An application of intervention mapping approach
}

\author{
Mehdi Mirzaei-Alavijeh ${ }^{1}$, Dilana Schaafsma², Behzad Karami-Matin ${ }^{3}$, Farzad Jalilian*1
}

Received: 19 Feb 2018

Published: 7 Aug 2019

\begin{abstract}
Background: Fecal occult blood test (FOBT) is one of the common screening tests for colorectal cancer. This study was designed to determine the socio-cognitive determinants related to FOBT uptake for colorectal cancer screening based on intervention mapping (IM).

Methods: A total of 500 individuals aged over 50 years were randomly selected to participate in this study in Kermanshah, Iran, in 2016. Data were collected by interviews based on a questionnaire and analyzed by SPSS16 using bivariate correlation, linear, and logistic regression models.

Results: Of the 500 respondents, 468 (93.6\%) signed the consent form and voluntarily participated in the study. Almost $11.1 \%$ of the participants had a history of FOBT uptake. Socio-cognitive variables accounted for $38 \%$ of the variation in the outcome measure of the intention to uptake FOBT. Perceived self-efficacy ( $\mathrm{OR}=3.345 \& 95 \% \mathrm{CI}: 1.342,8.339)$, perceived susceptibility $(\mathrm{OR}=2.204 \& 95 \%$ CI: $1.320,3.680)$, attitude $(\mathrm{OR}=1.674 \& 95 \% \mathrm{CI}: 1.270,2.137)$, and perceived severity $(\mathrm{OR}=1.457 \& 95 \% \mathrm{CI}: 0.954,2.224)$ were the strongest predictors of fecal occult blood test uptake.

Conclusion: IM-based analysis of behavior may provide insights to design interventions for modifying individuals' beliefs about the usefulness of FOBT uptake to prevent colorectal cancer.
\end{abstract}

Keywords: Early detection of cancer, Self-efficacy, Attitude, Disease susceptibility

Conflicts of Interest: None declared

Funding: Kermanshah University of Medical Sciences

*This work has been published underCC BY-NC-SA 1.0 license.

Copyright $₫$ Iran University of Medical Sciences

Cite this article as: Mirzaei-Alavijeh M, Schaafsma D, Karami-Matin B, Jalilian F. Socio-cognitive determinants of colorectal cancer screening uptake: An application of intervention mapping approach.Med J Islam Repub Iran. 2019(7 Aug);33:80. https://doi.org/10.47176/mjiri.33.80

\section{Introduction}

Cancer is one of the most important causes of morbidity and mortality worldwide and is the third most common cause of death in Iran $(1,2)$. CRC is the third most common cancer in Iranian women and fifth in men based on the Iranian Annual National Cancer Registration Report

\footnotetext{
Corresponding author: Dr Farzad Jalilian,f_jalilian@yahoo.com
}

1. Social Development \& Health Promotion Research Center, Health Institute, Kermanshah University of Medical Sciences, Kermanshah, Iran

2. Fontys University of Applied Sciences, Eindhoven, the Netherlands

3. Research Center for Environmental Determinants of Health, Health Institute, Kermanshah University of Medical Sciences, Kermanshah, Iran
(3). The reports have shown that 5000 individuals are annually diagnosed with CRC in Iran (4).

Screening programs are essential for early detection of CRC because of the high incidence and mortality of CRC (5). CRC is one of the best candidates for early cancer

$\uparrow$ What is "already known" in this topic:

Cancer screening-related behaviors, such as FOBT uptake, with the aim of early diagnosis of CRC is very useful in reducing the rate of cancer-related morbidity and mortality. Low CRC screening uptake is linked to low benefits of screening, fear of cancer, lack of knowledge towards screening behaviors, lack of susceptibility and severity about the side effects of CRC, and the low efficacy to uptake screening tests. Thus, providing information to promote CRC screening behaviors is vital to develop health promotion programs.

$\rightarrow$ What this article adds:

This research showed that females were more likely to uptake FOBT. Also, the findings indicated that modifying or improving individuals' positive beliefs towards FOBT uptake among at-risk groups can promote early detection behavior towards colorectal cancer. 
detection and screening; and the European code against cancer recommends a population-based approach for CRC screening (6). Several countries have introduced national programs of CRC screening (7). In Iran, all at-risk CRC groups are invited to complete a CRC screening test, such as faecal occult blood test (FOBT) (8). FOBT screening uptake is significantly related to reducing CRC mortality rates by detecting the disease at an earlier stage (9). Libby et al performed a population-based study on males and females aged 50-69 years in Scotland and reported that FOBT uptake reduced the overall CRC mortality by $10 \%$ and by $27 \%$ among those who returned a completed test kit (10). Other studies reported that annual FOBT uptake screening has been demonstrated to reduce $20 \%$ and $33 \%$ CRC incidence and mortality, respectively (11-13). Therefore, planning FOBT uptake screening programs for atrisk groups are now being implemented in numerous countries (7). Despite the clear benefits of screening, evidence indicates that CRC screening is low among at-risk groups (14). It seems necessary to develop programs that aim to encourage at-risk groups to participate in screening programs on a regular basis (15). In addition, human behavior is reflective of various determinants and acknowledging this causal relationship is crucial for understanding effective determinants (16). In the present study, to determine socio-cognitive determinants related to FOBT uptake for CRC screening, intervention mapping approach was used (IM); IM is an approach for developing theory and evidence-based health promotion programs and is composed of 6 steps, which enable planners to make evidence available in each step of program planning (17). Based on IM, in the first step, using relevant literature, the effective socio-cognitive determinants related to FOBT uptake were identified and the most important and the most changeable determinants were prioritized $(17,18)$. In addition, a comprehensive cancer preventative plan needs to focus on socio-cognitive predictors of these behaviors (19-21). Several studies indicated that low uptake in CRC screening has also been linked to low benefits of screening, fear of cancer, lack of knowledge towards screening behaviors, lack of susceptibility, severity about the side effects of $\mathrm{CRC}$, and low efficacy to take screening tests (22-26).

This IM-based study focused on determining sociocognitive determinants related to FOBT uptake for CRC screening in a sample of males and females aged over 50 years in the west of Iran.

\section{Methods \\ Participants and setting}

This cross sectional study was conducted among male and female participants older than 50 years in the west of Kermanshah province, Iran, during 2016. According to the findings of a pilot study, the sample size was calculated at $95 \%$ significance level and a sample of 500 was calculated. The following stages were done for data collection. First, different areas of the city were categorized based on the division of the geographical region. Next, for each geographical region, 1 health center was randomly selected (A total of 2 health centers were selected.).
Then, using simple random sampling method with proportion to size, the participants were selected from the medical records of the residents available at the centers for each cluster. The participants' postal address was documented, so they were interviewed at their home. Only those aged over 50 years who had no history of CRC were candidates to participate in the study. Finally, data were collected by interviewing the volunteers based on a questionnaire. The participants were assured that the gathered information will be used for scientific studies and remain confidential. Of the population of 500, a total of 468 $(93.6 \%)$ were voluntarily agreed to participate in the study.

\section{Measures}

The questionnaire included 3 sections. Prior to conducting the main project, a pilot study was conducted to assess the utility of the questionnaire, which was done among 30 participants who were similar to the participants of the main study. This was done to examine the clarity and comprehensiveness of various instruments and to collect data to estimate the internal consistency of the measures. Furthermore, content validity was confirmed by a group of experts $(2$ internists, 6 health education and promotion doctors, 2 health care services management doctors, and 2 public health experts).

\section{A: Demographics questionnaire}

The background variables assessed in this study included age (years), sex, marital status (single, married, widowed, divorced), and educational level (illiterate, primary school, secondary school, high school diploma, university degree).

\section{B: Questionnaire about CRC and FOBT general infor-} mation

The second section had 7 items about CRC general information: (1) having knowledge about what is CRC (yes, no); (2) family history of CRC (yes, no); (3) having knowledge about what is FOBT (yes, no); (4) FOBT uptake (yes, no); (5) the last FOBT uptake (less than 1 year, 1-2 years ago, 3-4 years ago, more than 5 years ago); (6) reason for not taking-up FOBT (I do not know what is FOBT; forgetting; fear of FOBT uptake; fear of results; not having enough time); and (7) what are the sources of your information about FOBT uptake (I did not have any information about it; physicians; family members; health care workers; Radio and TV; internet; newspapers \& scientific publications).

\section{C: Socio-cognitive determinants questionnaire}

The items on socio-cognitive determinants were developed based on standard questionnaires (22-26) and included 42 items under 8 constructs: (a) attitude; (b) subjective norms; (c) perceived self-efficacy; (d) behavioral intention; (e) perceived susceptibility; (f) perceived severity; (g) knowledge; and (h) perceived barriers. In the sociocognitive determinants questionnaire, 5 items evaluate attitude toward FOBT uptake (eg,"For me, FOBT uptake is unpleasant/pleasant."); 5 evaluate subjective norms toward FOBT uptake (eg," Most of my family and friends think that I should uptake FOBT to prevent CRC."); 2 measured perceived self-efficacy toward FOBT uptake 
(eg, "It is difficult for me to uptake FOBT."); 3 measure behavioral intention about FOBT uptake (eg, "I intend to uptake FOBT in this year."); 5 evaluate perceived susceptibility about the chance of being afflicted with CRC (eg, "It is likely that I will be afflicted with CRC in the future."); 5 measure perceived severity about side effects of CRC (eg, "CRC is a serious and dangerous disease."); 4 measure perceived barriers to FOBT uptake (eg, "FOBT uptake is too expensive for me"); and 14 items measure knowledge about the risk factors of CRC, such as physical inactivity, stress, etc.

A 5-point Likert scale, ranging from 1 (strongly disagree) to 5 (strongly agree) was used to measure participants' responses to the items of subjective norms, perceived self-efficacy, behavioral intention, perceived susceptibility, perceived severity, and perceived barriers. Attitude item was standardized to a 7-point Likert scale, ranging from 1 to 7 . Responses to the knowledge items were either yes or no or I don't know.

Using Cronbach's alpha coefficient, the estimated reliability for attitude $(\alpha=0.89)$, subjective norms $(a=0.74)$, perceived self-efficacy $(\alpha=0.62)$, behavioral intention $(\alpha=$ $0.70)$, perceived susceptibility ( $\alpha=0.71)$, perceived severity $(\alpha=0.75)$, and perceived barriers $(\alpha=0.74)$ were determined. Also, reliability coefficient for the knowledge scale was found to be 0.64 using the split-half method.

\section{Ethics statement}

Ethical approval for the research was obtained from the Ethical Committee of Kermanshah University of Medical Sciences (KUMS) (KUMS.REC.1394.269). The participants were informed about the aim and design of the study, participation was voluntary, and they were ensured that their identities and responses will be kept confidential.

\section{Statistical analysis}

Descriptive statistics were used to summarize and organize the data. Bivariate correlations were computed to determine the magnitude and direction of the associations between the socio-cognitive determinants. Multiple logistic regression analysis was performed to explain the sociodemographic characteristics and socio-cognitive de- terminants related to FOBT uptake (as a dependent variable). In addition, linear regressionmodel was performed to explain the intention to uptake FOBT as the outcome variable. Cronbach's alpha coefficient and split-half method were used to estimate the internal consistency of the various measures. Data entry and analyses were done using SPSS 16.

\section{Results}

The mean \pm SD age of the participants was $58.50 \pm 6.54$ years [95\% CI: 57.90, 59.09], ranging from 50 to 75 years. Of the participants, $52.6 \%(246 / 468)$ were male and $47.4 \%$ (222/468) were female, and 76.3\% (357/468) were married and 23.7\% (111/468) were single. Also, 61.1\% $(286 / 468)$ of them were illiterate and had primary school ( $>5$ grades of education) literacy, 26.5\% (124/468) did not have a high school diploma ( $>12$ grades), 9.8\% (46/468) had a high school diploma, and 2.6\% (12/468) had academic education. Moreover, $87.2 \%$ had health insurance, $4.7 \%$ (22/468) had knowledge about colorectal cancer, and $3.6 \%(17 / 468)$ reported a positive family history of CRC. Also, 11.1\% (52/468) of the participants had a history of FOBT uptake, and about $80.6 \%$ (377/468) of the participants stated that the most important reason for which they did not uptake FOBT was lack of knowledge. Logistic regression (backward stepwise method) was performed to explain the sociodemographic characteristics related to FOBT uptake (yes, no), and the best model was selected in the 4th step. Among the sociodemographic characteristics, age, sex, education level, and positive history of colorectal cancer were the most influential predictive factors related to FOBT uptake (Table 1).

Table 2 demonstrates the associations among the predictor variables based on bivariate. For example, intention of FOB uptake was associated with positive attitudes towards FOB uptake $(\mathrm{r}=0.476)$, subjective norms $(\mathrm{r}=0.387)$, perceived self-efficacy $(\mathrm{r}=0.384)$, perceived susceptibility $(\mathrm{r}=0.442)$, and perceived severity $(\mathrm{r}=0.259)$, while it was not correlated with perceived barrier $(\mathrm{r}=-0.078)$ and knowledge $(r=0.088)$.

The results of the logistic regression analysis (backward stepwise Wald) showed that the final model was suitable in the fourth step and that among the variables entered,

Table 1.Multiple logistic regression results for sociodemographic characteristics related to FOBT uptake (final model. Step 4)

\begin{tabular}{|c|c|c|c|c|}
\hline Variables & $\begin{array}{l}\text { Crude OR } \\
(95 \% \mathrm{CI})\end{array}$ & $\mathrm{p}$ & $\begin{array}{c}\text { Adjusted OR } \\
(95 \% \mathrm{CI})\end{array}$ & $\mathrm{p}$ \\
\hline \multicolumn{5}{|l|}{$\overline{\text { Age }}$} \\
\hline $50-59$ years & 1 & - & 1 & - \\
\hline $60-69$ years & $1.087(0.552-2.138)$ & 0.810 & $1.740(0.756-4.007)$ & 0.193 \\
\hline $70-75$ years & $2.935(1.306-6.598)$ & 0.009 & $4.136(1.513-11.302)$ & 0.006 \\
\hline \multicolumn{5}{|l|}{ Sex } \\
\hline Male & 1 & - & 1 & - \\
\hline Female & $1.903(1.054-3.437)$ & 0.033 & $2.773(1.325-5.805)$ & 0.007 \\
\hline \multicolumn{5}{|l|}{ Education } \\
\hline Illiterate and primary school & 1 & - & 1 & \\
\hline Secondary & $1.107(0.505-2.425)$ & 0.800 & $2.691(1.074-6.745)$ & 0.035 \\
\hline High school diploma & $6.730(3.173-14.277)$ & $<0.001$ & $15.418(6.002-39.606)$ & $<0.001$ \\
\hline Academic & $9.014(2.633-30.858)$ & $<0.001$ & $25.281(6.232-102.558)$ & $<0.001$ \\
\hline \multicolumn{5}{|c|}{ Positive history of colorectal cancer in family } \\
\hline No & 1 & - & 1 & - \\
\hline Yes & $4.802(1.697-13.593)$ & 0.003 & $4.292(1.309-14.076)$ & 0.016 \\
\hline
\end{tabular}


Table 2.Correlation of socio-cognitive variables based on bivariate correlation analysis

\begin{tabular}{|c|c|c|c|c|c|c|c|c|}
\hline & Mean (SD) & $\mathrm{X}^{1}$ & $\mathrm{X}^{2}$ & $\mathrm{X}^{3}$ & $\mathrm{X}^{4}$ & $\mathrm{X}^{5}$ & $\mathrm{X}^{6}$ & $\mathrm{X}^{7}$ \\
\hline $\mathrm{X}^{1}$. Attitude & $11.93(7.53)$ & 1 & & & & & & \\
\hline $\mathrm{X}^{2}$. Subjective Norms & $7.35(2.85)$ & $0.214^{* *}$ & 1 & & & & & \\
\hline $\mathrm{X}^{3}$. Self-efficacy & $5.97(1.45)$ & $0.258^{* *}$ & $0.237^{* *}$ & 1 & & & & \\
\hline $\mathrm{X}^{4}$. Perceived Susceptibility & $13.67(3.34)$ & $0.596^{* *}$ & $0.178^{* *}$ & $0.222^{* *}$ & 1 & & & \\
\hline $\mathrm{X}^{5}$. Perceived Severity & $16.07(4.06)$ & $0.359^{* *}$ & $0.092^{*}$ & $0.210^{* *}$ & $0.601^{* *}$ & 1 & & \\
\hline $\mathrm{X}^{6}$. Perceived Barriers & $10.17(2.61)$ & $-0.178^{* *}$ & -0.038 & -0.073 & $-0.106^{*}$ & $-0.111^{*}$ & 1 & \\
\hline $\mathrm{X}^{7}$. Knowledge & $5.87(2.74)$ & $0.213^{* *}$ & 0.074 & $0.138^{* *}$ & $0.264^{* *}$ & $0.161^{* *}$ & $-0.092^{*}$ & 1 \\
\hline $\mathrm{X}^{8}$. Behavioural Intention & $7.58(2.08)$ & $0.476^{* *}$ & $0.387^{* *}$ & $0.384^{* *}$ & $0.442^{* *}$ & $0.256^{* *}$ & -0.078 & 0.088 \\
\hline
\end{tabular}

$* \mathrm{p}<0.05, * * \mathrm{p}<0.01$

Table 3. Results of socio-cognitive variables related to FOBT uptake based on multiple logistic regression

\begin{tabular}{lccc}
\hline Variables & Odds Ratio & \multicolumn{2}{c}{$95 \%$ Confidence Intervals } \\
\cline { 3 - 4 } & & Lower & Upper \\
Attitude & 1.647 & 1.270 & 2.137 \\
Self-efficacy & 3.345 & 1.342 & 8.339 \\
Susceptibility & 2.204 & 1.320 & 3.680 \\
Severity & 1.457 & 0.954 & 0.001 \\
\hline
\end{tabular}

Table 4.Linear regression results with the intention to uptake FOBT as an outcome variable

\begin{tabular}{|c|c|c|c|}
\hline Variable & $\mathrm{B}$ & $\mathrm{T}$ & $\mathrm{p}$ \\
\hline Attitude & 0.253 & 5.467 & $<0.001$ \\
\hline Subjective norms & 0.245 & 6.439 & $<0.001$ \\
\hline Self-efficacy & 0.205 & 5.298 & $<0.001$ \\
\hline Susceptibility & 0.206 & 4.530 & $<0.001$ \\
\hline
\end{tabular}

Adjusted $\mathrm{R}$ squared $=0.38, \mathrm{~F}=72.828, \mathrm{p}<0.001$

perceived self-efficacy $(\mathrm{OR}=3.345 \&$ $\& 5 \% \mathrm{CI}: 1.342$ $8.339)$, perceived susceptibility $(\mathrm{OR}=2.204 \& 95 \% \mathrm{CI}$ : $1.320,3.680)$, attitude $(\mathrm{OR}=1.674 \& 95 \% \mathrm{CI}: 1.270$, 2.137), and perceived severity $(\mathrm{OR}=1.457 \& 95 \% \mathrm{CI}$ : $0.954,2.224)$ were, respectively, the most strong predictors of FOBT uptake (Table 3 ).

A hierarchical multiple regression analysis was performed to explain the variation in intention to uptake FOBT. Socio-cognitive variables were accounted for $38 \%$ of the variation in intention to uptake FOBT $(\mathrm{p}<0.001)$ (Table 4).

\section{Discussion}

This study aimed to determine the socio-cognitive determinants related to FOBT uptake for colorectal cancer screening. The intervention mapping approach was conducted for need assessment among participants. The results of this study suggest that socio-cognitive variables accounted for $38 \%$ of the variation in the outcome measure of the intention to uptake FOBT. Furthermore, perceived self-efficacy, perceived susceptibility, and attitude were the strongest predictors of FOBT uptake, respectively.

According to the results, $11.1 \%$ of the participants had history of FOBT uptake at least once. Ricardo-Rodrigues et al performed a longitudinal study among patients aged 50-69 years in Spain and reported that the rate of FOBT uptake was $4.23 \%$ in 2009 , which increased to $7.74 \%$ in 2011 (27). Yamamichi et al reported that the screening rate for CRC was $24.9 \%$ in Japan (28). Lo et al, in their study among 1309 individuals aged 60 to 70 years in the UK, reported that $69.4 \%$ of their participants had an experience of at least $1 \mathrm{CRC}$ screening (29). One of the aims of the Healthy People 2020 initiative for cancer screening is to reach a target statistic of $70 \%$ to cover CRC screening (30). Considering the aims of the Healthy People 2020 for covering CRC screening, FOBT uptake is much lower among Iranian people.

The findings of this study indicated that women had a greater probability for FOBT uptake. This result is not similar to the results reported by Morris et al (31). In addition, Lo et al, in their study among participants aged 60 70 years living in England, found no differences in CRC screening uptake by gender (29). However, Symonds et al, who performed a research in South Australia from 2008 to 2013, reported that higher positive rate in fecal immunochemical test (FIT) was independently associated with males (22). Furthermore, Ricardo-Rodrigues et al, in their study in Spain, stated that males had greater adherence to FOBT uptake compared to females (27). In early cancer detection behaviors, sex differences could begin to make a significant contribution to mortality differentials (30). These results may, therefore, have important implications for offering CRC screening programs.

The participants aged 70 years and older had FOBT uptake much more than the younger participants. The findings of this study were similar to other studies $(33,34)$. This may be due to the fact that younger people may think they are at a lower risk of colorectal cancer.

This study also revealed that FOBT uptake is often related to high level of education, as those who had a high school diploma or an academic degree were more likely to uptake FOBT, which was similar to other studies (35-37). Perhaps knowledge of the risk symptoms caused the person to adopt screening behaviors. Therefore, developing and implementing interventions for groups with lower education levels is of high importance.

Another finding of the present study was the influential predictive factor of positive history of CRCrelated to FOBT uptake. Similar studies confirmed this finding (27). It seems that those who had positive history of CRC were more willing to uptake cancer screening behaviors, as they thought their perceived risk was high. 
According to the results, physicians had an important role in providing FOBT-related information to participants. Similar studies confirmed this finding $(38,39)$ and confirmed the effectiveness of the physicians' role in persuading the participants to take part in cancer screening programs. Sung et al (39), in their study, indicated that physicians' recommendation and insurance coverage were the most important cues to action and can increase CRC screening behaviors.

Socio-cognitive determinants suggest that decisions to engage in health promoting behaviors are influenced by psychological determinants (40-42). The main aim of this study was to determine socio-cognitive determinants related to FOBT uptake among a population of Iranian males and females aged over 50 years.

Also, the results suggested that the following 4 sociocognitive determinants were related to the FOBT uptake among the Iranian middle-aged and elderly: (1) perceived self-efficacy towards FOBT uptake;(2) perceived susceptibility towards possibility of getting CRC;(3) positive attitude towards FOBT uptake; and (4) perceived severity towards side effect of CRC. Consistent with previous research (21-26), the findings of this study showed a connection between the social cognitive determinants and CRC screening behaviors.

Also, perceived self-efficacy was found to be a strong predictor for FOBT uptake. Hawley et al performed a research on 1224 individuals aged 50-70 years who were at average CRC risk and overdue for screening in KelseySeybold Clinic, the largest multispecialty medical organization in Houston, Texas. They reported that preference for FOBT uptake was associated with self-efficacy for doing FOBT (43). Furthermore, Von Wagner et al indicated that higher perceived self-efficacy leads to greater participation in CRC screening (25). It seems that designing health promotion programs for at-risk groups to increase self-efficacy about what FOBT test entails may increase their self-efficacy and may be useful to increase FOBT uptake.

Based on the findings of the present study, people who did perceive positive attitude to FOBT uptake screening were more likely to participate in screening. Gregory et al indicated that an intervention that could successfully modify positive attitude of CRC screening behaviors should lead to an increase in the participation rates for CRC screening (41).

Moreover, in this study, it was found that perceived susceptibility towards possibility of getting CRC and perceived severity towards side effects of CRC were 2 important factors that predict FOBT uptake. Several studies reported that changes in perceived susceptibility and severity were mediators for promoting effectiveness of CRC screening promotion programs $(44,45)$.

Finally, the findings showed they were accounted for $38 \%$ of the variation in intention to FOBT uptake. Sieverding et al studied social norms screening behaviors among 2426 males, with the mean age of 56.3 years, in Germany and reported that attitude, subjective norms, and perceived behavior control variables predicted $49 \%$ of the variance in behavioral intention for screening tests (46).
Consequently, the results confirmed that IM is a suitable theoretical basis for the need assessment of CRC screening promotion programs for high-risk groups.

\section{Conclusion}

In this study, it was found that females were more likely to uptake FOBT. The results of this study may be useful for guiding developers and implementers to develop and implement effective programs to promote FOBT uptake among the middle-aged and the elderly. Thus, IM-based analysis of behavior may provide insights for designing interventions to modify individuals' beliefs about the usefulness of FOBT uptake to prevent colorectal cancer. Policymakers and health care workers can use these results to design FOBT promotion programs.

\section{Limitations}

This study had several advantages, such as applying the above-mentioned theories and gathering data directly by interviewing the participants at their homes for their convenience. The results reported in this research had some limitations. First, data collection was based on selfreporting, which always has the risk of recall bias. The second limitation was that data were collected only from the sample of Iranian middle-age and elderly people in a small city in West of Iran, and thus the results could not be generalized to other middle-aged and elderly population in Iran. The third limitation was that the history of FOBT uptake was investigated using yes-no scale. Finally, the internal consistency of the questionnaire was relatively low $(\alpha=0.62)$ for perceived self-efficacy and $(\alpha=0.64)$ knowledge.

\section{Acknowledgements}

This article is part of a research project supported by Research Center for Environmental Determinants of Health in KUMS, Kermanshah, Iran. We would like to thank the research deputy of KUMS for funding this study.

\section{Funding}

The authors acknowledge and appreciate the funding provided by Kermanshah University of Medical Sciences. The funder had no role in designing the study, in collection, analysis, and interpretation of data, or in writing the manuscript.

\section{Conflict of Interests}

The authors declare that they have no competing interests.

\section{References}

1. Rafiemanesh H, Pakzad R, Abedi M, Kor Y, Moludi J, Towhidi F, Makhsosi BR, Salehiniya H. Colorectal cancer in Iran: Epidemiology and morphology trends. EXCLI J. 2016;15:738

2. Pourhoseingholi MA, Zali MR. Colorectal cancer screening: Time for action in Iran. World J Gastrointest Oncol. 2012; 4(4):82.

3. Siegel RL, Miller KD, Fedewa SA, Ahnen DJ, Meester RG, Barzi A, Jemal A. Colorectal cancer statistics, 2017. CA Cancer J Clin. 2017;67(3):177-93

4. Azadeh S, Moghimi-Dehkordi B, Fatem SR, Pourhoseingholi MA, 
Ghiasi S, Zali MR. Colorectal cancer in Iran: an epidemiological study. Asian Pac. J. Cancer Prev. 2008;9(1):123-6.

5. O'Carroll RE, Chambers JA, Brownlee L, Libby G, Steele RJ. Anticipated regret to increase uptake of colorectal cancer screening (ARTICS): a randomized controlled trial. Soc Sci Med. 2015;142:118-27

6. Van Roosbroeck S, Hoeck S, Van Hal G. Population-based screening for colorectal cancer using an immunochemical faecal occult blood test: a comparison of two invitation strategies. Cancer Epidemiol. 2012; 36(5):e317-24

7. Garborg K, Holme Ø, Løberg M, Kalager M, Adami HO, Bretthauer M. Current status of screening for colorectal cancer. Ann Oncol. 2013;24(8):1963-72.

8. Ramezani Doroh V, Delavari A, Yaseri M, Emamgholipour Sefiddashti $\bar{S}$, Akbarisari A. Preferences of Iranian average risk population for colorectal cancer screening tests. Int J Health Care Qual Assur. 2019; 32(4):677-87.

9. Todorov K, Wilson C, Sharplin G, Corsini N. Faecal occult blood testing (FOBT)-based colorectal cancer screening trends and predictors of non-use: findings from the South Australian setting and implications for increasing FOBT uptake. Aust Health Rev. 2018; 42(1):45-52.

10. Libby G, Brewster DH, McClements PL, Carey FA, Black RJ, Birrell $\mathrm{J}$, et al. The impact of population-based faecal occult blood test screening on colorectal cancer mortality: a matched cohort study. Br J Cancer. 2012;107(2):255-259.

11. Sung JJ, Ng SC, Chan FK, Chiu HM, Kim HS, Matsuda T, et al. An updated Asia Pacific Consensus Recommendations on colorectal cancer screening. Gut. 2015;64(1):121-32

12. Zauber AG. The impact of screening on colorectal cancer mortality and incidence: has it really made a difference?. Dig Dis Sci. 2015; 60(3):681-91.

13. Zorzi M, Fedeli U, Schievano E, Bovo E, Guzzinati S, Baracco S, Fedato C, Saugo M, Dei Tos AP. Impact on colorectal cancer mortality of screening programmes based on the faecal immunochemical test. Gut. 2015;64(5):784-90

14. Symonds EL, Cock C, Meng R, Cole SR, Fraser RJ, Young GP. Uptake of a colorectal cancer screening blood test in people with elevated risk for cancer who cannot or will not complete a faecal occult blood test. Eur J Cancer Prev. 2018;27(5):425-32.

15.Zavoral M, Suchanek S, Majek O, Fric P, Minarikova P, Minarik M, Seifert B, Dusek L. Colorectal cancer screening: 20 years of development and recent progress. World J Gastroenterol. 2014;20(14):3825.

16. Steinmetz H, Knappstein M, Ajzen I, Schmidt P, Kabst R. How effective are behavior change interventions based on the theory of planned behavior? A three-level meta-analysis. Z Psychol. 2016; 224(3):216.

17. Kok G. A practical guide to effective behavior change: How to apply theory-and evidence-based behavior change methods in an intervention. Health Psychol Rev. 2014;16(5):156-70.

18. Mirzaei-Alavijeh M, Kok G, Niknami S, Motlagh ME. Family-based cognitive factors effective on preventing the onset of substance use in Iranian society's children: applying the intervention mapping protocol. Acta Medica Mediterranea. 2016;32:1015-20.

19. Lamort $\square$ Bouché M, Sarnin P, Kok G, Rouat S, Péron J, Letrilliart L, Fassier JB. Interventions developed with the Intervention Mapping protocol in the field of cancer: a systematic review. Psycho $\square$ oncology. 2018; 27(4):1138-49.

20. Kok G, Gottlieb NH, Peters GJ, Mullen PD, Parcel GS, Ruiter RA, Fernández ME, Markham C, Bartholomew LK. A taxonomy of behaviour change methods: an Intervention Mapping approach. Health Psychol Rev. 2016; 10(3):297-312.

21. Hosseini SN, Alavijeh MM, Matin BK, Hamzeh B, Ashtarian H, Jalilian F. Locus of control or self-esteem; Which one is the best predictor of academic achievement in Iranian college students. Iran J Psychiatry Behav Sci. 2016;10(1): e2602.

22. Symonds EL, Osborne JM, Cole SR, Bampton PA, Fraser RJ, Young GP. Factors affecting faecal immunochemical test positive rates: demographic, pathological, behavioural and environmental variables. J Med Screen 2015;22:187-93.

23. Taniguchi T, Hirai K, Harada K, Ishikawa Y, Nagatsuka M, Fukuyoshi J, et al. The relationship between obtaining fecal occult blood test and beliefs regarding testing among Japanese. Health Psychol Behav Med. 2015;3:251-62.

24. Javanparast S, Ward P, Young G, Wilson C, Carter S, Misan G, Cole S, Jiwa M, Tsourtos G, Martini A, Gill T. How equitable are colorectal cancer screening programs which include FOBTs? A review of quali- tative and quantitative studies. Prev Med. 2010;50(4):165-72.

25. Von Wagner C, Semmler C, Good A, Wardle J. Health literacy and self-efficacy for participating in colorectal cancer screening: the role of information processing. Patient Educ Couns. 2009; 75(3):352-7.

26. Courtney RJ, Paul CL, Sanson-Fisher RW, Carey ML, Macrae FA, Yoong SL. Community approaches to increasing colorectal screening uptake: a review of the methodological quality and strength of current evidence. Cancer Forum 2012;36: 27-35.

27. Ricardo-Rodrigues I, Jiménez-García R, Hernández-Barrera V, Carrasco-Garrido P, Jiménez-Trujillo I, López-de-Andrés A. Adherence to and predictors of participation in colorectal cancer screening with faecal occult blood testing in Spain, 2009-2011. Eur J Cancer Prev. 2015;24(4):305-12.

28. Yamamichi J, Seto K, Hinotsu S, Nagata K, Kobayashi Y, Urushihara H, Kawakami K. Patterns and Trends in Diagnostic Tests Used for Detection of Colorectal Cancer after Screening with the Immunochemical Fecal Occult Blood Test in Japan. Open J Clin Diagn. 2015;5(03):107.

29. Lo SH, Waller J, Vrinten C, Kobayashi L, von Wagner C. Social cognitive mediators of socio-demographic differences in colorectal cancer screening uptake. Biomed Res Int. 2015:1-9.

30. Alford SH, Leadbetter S, Rodriguez JL, Hawkins NA, Scholl LE, Peipins LA. Cancer screening among a population-based sample of insured women. Prev Med Rep. 2015;2:15-20.

31. Morris S, Baio G, Kendall E, Von Wagner C, Wardle J, Atkin W, et al .Socioeconomic variation in uptake of colonoscopy following a positive faecal occult blood test result: a retrospective analysis of the NHS Bowel Cancer Screening Programme. Br J Cancer 2012; 107:765-773

32. Evans RE, Brotherstone H, Miles A, Wardle J. Gender differences in early detection of cancer. J Mens Health Gend. 2005; 2(2):209-17.

33. Power E, Miles A, Von Wagner C, Robb K, Wardle J. Uptake of colorectal cancer screening: system, provider and individual factors and strategies to improve participation. Future Oncol. 2009; 5(9):137188 .

34. Gimeno Garcia AZ. Factors influencing colorectal cancer screening participation. Gastroenterol Res Pract. 2012:1-8

35. Gimeno-García AZ, Quintero E, Nicolás-Pérez D, Parra-Blanco A, Jiménez A. Colorectal cancer screening in a Spanish population. Med Clin. 2009;133(19):736-40.

36. Meissner HI, Breen N, Klabunde CN, Vernon SW. Patterns of colorectal cancer screening uptake among men and women in the United States. Cancer Epidemiol Biomarkers Prev. 2006; 15(2):389-94.

37. Sun WY, Basch CE, Wolf RL, Li XJ. Factors associated with colorectal cancer screening among Chinese-Americans. Prev Med. 2004;39(2):323-9.

38. Arnold CL, Rademaker A, Liu D, Davis TC. Changes in colorectal cancer screening knowledge, behavior, beliefs, self-efficacy, and barriers among community health clinic patients after a health literacy intervention. J Community Med Health Educ. 2017; 7.

39. Sung JJ, Choi SY, Chan FK, Ching JY, Lau JT, Griffiths S. Obstacles to colorectal cancer screening in Chinese: a study based on the health belief model. Am J Gastroenterol. 2008; 103:974

40. Mirzaei-Alavijeh M, Ahmadi-Jouybari T, Vaezi M, Jalilian F. Prevalence, Cognitive and Socio-Demographic Determinants of Prostate Cancer Screening. Asian Pac. J. Cancer Prev. 2018; 19(4):1041-6

41. Gregory TA, Wilson C, Duncan A, Turnbull D, Cole SR, Young G. Demographic, social cognitive and social ecological predictors of intention and participation in screening for colorectal cancer. BMC Public Health. 2011;11(1):38

42. Mirzaei-Alavijeh M, Ghorbani P, Jalilian F. Socio-cognitive determinants of the mammography screening uptake among Iranian women. Asian Pac. J. Cancer Prev. 2018;19(5):1351-5.

43. Hawley ST, McQueen A, Bartholomew LK, Greisinger AJ, Coan SP, Myers R, et al.Preferences for colorectal cancer screening tests and screening test use in a large multispecialty primary care practice. Cancer. 2012;118:2726-34

44. Braun KL, Fong M, Kaanoi ME, Kamaka ML, Gotay CC. Testing a culturally appropriate, theory-based intervention to improve colorectal cancer screening among Native Hawaiians. Prev Med. 2005;40(6):619 27.

45. Bae N, Park S, Lim S. Factors associated with adherence to fecal occult blood testing for colorectal cancer screening among adults in the Republic of Korea. Eur J Oncol Nurs. 2014; 18(1):72-7.

46. Sieverding M, Matterne U, Ciccarello L. What role do social norms play in the context of men's cancer screening intention and behavior? 
Application of an extended theory of planned behavior. Health Psycho. 2010;29(1):72. 